\title{
Cluster ensembles, quantization and the dilogarithm II: The intertwiner
}

\author{
V.V. Fock, A. B. Goncharov \\ To Yuri Ivanovich Manin for his 70th birthday
}

\section{Contents}

1 Introduction

2 Cluster ensembles 3

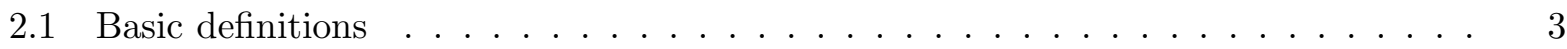

2.2 Connections between quantum $\mathcal{X}$-varieties . . . . . . . . . . . . 4

3 Motivation: *-quantization of cluster $\mathcal{X}$-varieties 6

$3.1 *$-quantization of the space $\mathcal{X}^{+}$via the quantum logarithm $\ldots \ldots \ldots$

3.2 Modular double of a cluster $\mathcal{X}$-variety and $*$-quantization of the space $\mathcal{X}^{+} \ldots \ldots$

4 The intertwiner $\quad \mathbf{1 0}$

4.1 A bimodule structure on functions on the $\mathcal{A}$-space . . . . . . . . . . . . . . . 10

4.2 The intertwiner via the quantum dilogarithm ................ 11

5 The quantum logarithm and dilogarithm functions

\section{Introduction}

Abstract. This paper is the second part of our preprint "Cluster ensembles, quantization and the dilogarithm" [FG2]. Its main result is a construction, by means of the quantum dilogarithm, of certain intertwiner operators, which play the crucial role in the quantization of the cluster $\mathcal{X}$-varieties and construction of the corresponding canonical representation.

Cluster ensemble (loc. cit.) is a pair $(\mathcal{A}, \mathcal{X})$ of schemes over $\mathbb{Z}$, called cluster $\mathcal{A}$ - and $\mathcal{X}$-varieties, related by a map $p: \mathcal{A} \longrightarrow \mathcal{X}$. The ring of regular functions on the cluster $\mathcal{A}$-variety is the upper cluster algebra $[\mathrm{BFZ}]$.

Cluster $\mathcal{A}$ - and $\mathcal{X}$-varieties are glued from families of coordinatized split algebraic tori by means of certain subtraction free rational transformations. In particular it makes sense to consider the spaces of their positive real points, denoted by $\mathcal{A}^{+}$and $\mathcal{X}^{+}$.

The cluster $\mathcal{X}$-variety has a Poisson structure, given in any cluster coordinate system $\left\{X_{i}\right\}$ by

$$
\left\{X_{i}, X_{j}\right\}=\widehat{\varepsilon}_{i j} X_{i} X_{j}, \quad \widehat{\varepsilon}_{i j} \in \mathbb{Z}
$$


The Poisson tensor $\widehat{\varepsilon}_{i j}$ depends on the choice of coordinate system. There is a canonical noncommutative deformation $\mathcal{X}_{q}$ of the cluster $\mathcal{X}$-variety in the direction of this Poisson structure ([FG2]).

The gluing procedure underlying the definition of a cluster variety can be understood as a functor from a certain groupoid, called the cluster modular groupid $\mathcal{G}$, to a category of commutative algebras.

In Section 3 we suggest a $*$-quantization of the cluster $\mathcal{X}$-variety, understood as a functor from the gropoid $\mathcal{G}$ to the category of non-commutative topological $*$-algebras. More precisely, the coordinate systems on cluster varieties are parametrised by the objects $\mathbf{i}$ of the groupoid $\mathcal{G}$, called seeds. To each seed $\mathbf{i}$ we assign two coordinatized tori, $\mathcal{A}_{\mathbf{i}}$ and $\mathcal{X}_{\mathbf{i}}$. The algebra of the smooth functions on the latter admits a canonical $\hbar$-deformation, given by a topological Heisenberg $*-$ algebra $\mathcal{H}_{\mathbf{i}}^{\hbar}$. So to define a functor we need to relate these algebras for different seeds. We write the formulas relating the generators of the algebras $\mathcal{H}_{\mathbf{i}}^{\hbar}$, but do not specify the category of topological *-algebras. As a result, the $*$-quantization of the cluster $\mathcal{X}$-variety serves only as a motivation, and we state in Sections 3 Claims instead of Theorems when those unspecified topological algebras enter the formulations. Hopefully there will be a precise version of Section 3. However the rest of the paper does not depend on that, while motivations given in Section 3 clarify what we do next.

In Section 4 we proceed to a construction of the canonical unitary projective representation of the modular gropoid. It is realized in the Hilbert space $L^{2}\left(\mathcal{A}^{+}\right)$assigned to the set of positive real points of the cluster $\mathcal{A}$-variety.

For each seed $\mathbf{i}$ there is a Hilbert space $L^{2}\left(\mathcal{A}_{\mathbf{i}}^{+}\right)$(which is canonically identified with $L^{2}\left(\mathcal{A}^{+}\right)$). In Section 4.1 the Heisenberg $*$-algebra $\mathcal{H}_{\mathbf{i}}^{\hbar}$ is represented in $L^{2}\left(\mathcal{A}_{\mathbf{i}}^{+}\right)$. In fact a bigger algebra the chiral double $\mathcal{H}_{\mathrm{i}}^{\hbar} \otimes \mathcal{H}_{\mathbf{i}^{\circ}}^{\hbar}$ of the Heisenberg $*$-algebra - acts on the same Hilbert space.

The morphisms in the groupoid $\mathcal{G}$ are defined as compositions of certain elementary ones, called mutations and symmetries. Given a mutation $\mathbf{i} \rightarrow \mathbf{i}^{\prime}$ we construct a unitary operator

$$
\mathbf{K}_{\mathbf{i} \rightarrow \mathbf{i}^{\prime}}: L^{2}\left(\mathcal{A}_{\mathbf{i}}^{+}\right) \longrightarrow L^{2}\left(\mathcal{A}_{\mathbf{i}^{\prime}}^{+}\right) .
$$

It intertwines the actions of the Heisenberg *-algebras related to the seeds $\mathbf{i}$ and $\mathbf{i} 1$ 1. (Similar itertwiners for symmetries are rather tautological). This construction is the main result of the paper. The operator $\mathbf{K}_{\mathbf{i} \rightarrow \mathbf{i}^{\prime}}$ is characterised by its intertwining property uniquely up to a constant.

Certain compositions of mutations and symmetries are identity morphisms in the groupoid $\mathcal{G}$. So to get a representation of the modular groupoid we have to show that the corresponding compositions of the intertiners $\mathbf{K}_{\mathbf{i} \rightarrow \mathbf{i}^{\prime}}$ are multiples of the identity operators. This is a rather difficult problem. It is solved in [FG3], where we give another construction of the intertwiner and introduce the geometric object reflecting its properties, the cluster double. Alltogether the intertwiners give rise to a unitary projective reprersentation of the cluster modular groupoid.

The paper is organised as follows: the essential for us properties of the quantum logarithm and dilogarithm are collected, without proofs in Section 5. The proofs and more of the properties of these functions can be found in Section 4 of [FG3]. In Section 2.1 we recall, for the convinience of the reader, basic definitions/facts about cluster ensembles. Claim 3.6 delivers a quantization of the space of real positive points of the cluster $\mathcal{X}$-variety. The main results of this paper is Theorem 4.3 providing an explicit formula for the intertwiner $\mathbf{K}_{\mathbf{i} \rightarrow \mathbf{i}^{\prime}}$.

\footnotetext{
${ }^{1}$ The precise meaning we put into "intertwining" is clear from the computation carried out in the proof: we deal with the genrators of the Heisenbrg algebra only, and thus are not concerned with the nature of the topological completion, which was left unspecified in Section 3. For a different approach see [FG3].
} 
Acknowledgments. V.F. was supported by the grants CRDF 2622; 2660. A.G. was supported by the NSF grants DMS-0099390 and DMS-0400449.

\section{Cluster ensembles}

\subsection{Basic definitions}

A seed $\mathbf{i}$ is a triple $(I, \varepsilon, d)$, where $I$ is a finite set, $\varepsilon$ is a matrix $\varepsilon_{i j}, i, j \in I$, with $\varepsilon_{i j} \in \mathbb{Z}$, and $d=\left\{d_{i}\right\}, i \in I$, are positive integers, such that the matrix $\widehat{\varepsilon}_{i j}:=\varepsilon_{i j} d_{j}^{-1}$ is skew-symmetric.

For a seed $\mathbf{i}$ we assign a torus $\mathcal{X}_{\mathbf{i}}=\left(\mathbb{G}_{m}\right)^{I}$ with the coordinates $\left\{X_{i} \mid i \in I\right\}$ on the factors. and a Poisson structure given by

$$
\left\{X_{i}, X_{j}\right\}=\widehat{\varepsilon}_{i j} X_{i} X_{j} .
$$

Let $\mathbf{i}=(I, \varepsilon, d)$ and $\mathbf{i}^{\prime}=\left(I^{\prime}, \varepsilon^{\prime}, d^{\prime}\right)$ be two seeds, and $k \in I$. A mutation in the direction $k \in I$ is an isomorphism $\mu_{k}: I \rightarrow I^{\prime}$ such that $d_{\mu_{k}(i)}^{\prime}=d_{i}$, and

$$
\varepsilon_{\mu_{k}(i) \mu_{k}(j)}^{\prime}= \begin{cases}-\varepsilon_{i j} & \text { if } i=k \text { or } j=k \\ \varepsilon_{i j} & \text { if } \varepsilon_{i k} \varepsilon_{k j} \leq 0 \\ \varepsilon_{i j}+\left|\varepsilon_{i k}\right| \varepsilon_{k j} & \text { if } \varepsilon_{i k} \varepsilon_{k j}>0\end{cases}
$$

A symmetry of a seed $\mathbf{i}=(I, \varepsilon, d)$ is an automorphism $\sigma$ of the set $I$ preserving the matrix $\varepsilon$ and the numbers $d_{i}$. Symmetries and mutations induce rational maps between the corresponding seed $\mathcal{X}$-tori, denoted by the same symbols $\mu_{k}$ and $\sigma$ and given by the formulae $\sigma^{*} X_{\sigma(i)}=X_{i}$ and

$$
\mu_{k(i)}^{*} X_{\mu_{k}(i)}= \begin{cases}X_{k}^{-1} & \text { if } \quad i=k \\ X_{i}\left(1+X_{k}^{-\operatorname{sgn}\left(\varepsilon_{\mathrm{ik}}\right)}\right)^{-\varepsilon_{i k}} & \text { if } \quad i \neq k .\end{cases}
$$

A seed cluster transformation is a composition of symmetries and mutations. Two seeds are equivalent if related by a cluster transformation. The equivalence class of a seed $\mathbf{i}$ is denoted by $|\mathbf{i}|$. A seed cluster transformation induces a rational map between the two seed $\mathcal{X}$-tori, called a cluster transformation map.

A cluster $\mathcal{X}_{\text {-variety }} \mathcal{X}_{|\mathbf{i}|}$ is a scheme over $\mathbb{Z}$ obtained by gluing the seed $\mathcal{X}$-tori for the seeds equivalent to a given seed $\mathbf{i}$ via the cluster transformation maps, and then taking the affine closure. Every seed provides a cluster $\mathcal{X}$-variety with a rational coordinate system. Its coordinates are called cluster coordinates. Cluster transformation maps preserve the Poisson structure. Thus a cluster $\mathcal{X}$-variety has a canonical Poisson structure.

The cluster $\mathcal{A}$-varieties. Given a seed $\mathbf{i}$, define a seed $\mathcal{A}$-torus $\mathcal{A}_{\mathbf{i}}:=\left(\mathbb{G}_{m}\right)^{I}$ with the standard standard coordinates $\left\{A_{i} \mid i \in I\right\}$ on the factors. Symmetries and mutations give rise to birational maps between the seed $\mathcal{A}$-tori, given by $\sigma^{*} A_{\sigma(i)}=A_{i}$ and

$$
\mu_{k(i)}^{*} A_{\mu_{k}(i)}= \begin{cases}A_{i} & \text { if } i \neq k, \\ A_{k}^{-1}\left(\prod_{i \mid \varepsilon_{k i}>0} A_{i}^{\varepsilon_{k i}}+\prod_{i \mid \varepsilon_{k i}<0} A_{i}^{-\varepsilon_{k i}}\right) & \text { if } i=k .\end{cases}
$$

The cluster $\mathcal{A}$-variety $\mathcal{A}_{|\mathrm{i}|}$ is a scheme over $\mathbb{Z}$ obtained by gluing all seed $\mathcal{A}$-tori for the seeds equivalent to a given seed $\mathbf{i}$ using the above birational isomorphisms, and taking the affine closure.

There is a map $p: \mathcal{A} \longrightarrow \mathcal{X}$, given in every cluster coordinate system by $p^{*} X_{k}=\prod_{I \in I} A_{i}^{\varepsilon_{k i}}$. 
Cluster $\mathcal{A}$ - and $\mathcal{X}$-varieties have canonical positive atlases, so it makes sense to consider the sets of their real positive points, denoted $\mathcal{A}^{+}$and $\mathcal{X}^{+}$.

The cluster modular groupoid. Seed cluster transformations inducing the same map of the seed $\mathcal{A}$-tori are called trivial seed cluster transformations. The cluster modular groupoid $\mathcal{G}_{|\mathbf{i}|}$ is a groupoid whose objects are seeds equivalent to a given seed $\mathbf{i}$, and $\operatorname{Hom}\left(\mathbf{i}, \mathbf{i}^{\prime}\right)$ is the set of all seed cluster transformations from $\mathbf{i}$ to $\mathbf{i}^{\prime}$ modulo the trivial ones. Given a seed $\mathbf{i}$, the cluster mapping class group $\Gamma_{\mathbf{i}}$ is the automorphism group of the object $\mathbf{i}$ of $\mathcal{G}_{|\mathbf{i}|}$. The group $\Gamma_{\mathbf{i}}$ acts by automorphisms of the cluster $\mathcal{A}$-variety.

The quantum space $\mathcal{X}_{q}$. It is a canonical non-commutative $q$-deformation of the cluster $\mathcal{X}$ variety defined in Section 3 of [FG2]. We start from the seed quantum torus algebra $\mathrm{T}_{\mathbf{i}}^{q}$, defined as an associative $*$-algebra with generators $X_{i}^{ \pm 1}, i \in I$ and $q^{ \pm 1}$ and relations

$$
q^{-\widehat{\varepsilon}_{i j}} X_{i} X_{j}=q^{-\widehat{\varepsilon}_{j i}} X_{j} X_{i}, \quad * X_{i}=X_{i}, \quad * q=q^{-1} .
$$

Let QTor* be a category whose objects are quantum torus algebras and morphisms are $*$-homomorphisms of their fraction fields. The quantum space $\mathcal{X}_{q}$ is understood as a contravariant functor

$$
\eta^{q}: \text { The modular groupoid } \mathcal{G}_{|\mathbf{i}|} \longrightarrow \text { QTor* }
$$

It assigns to a seed $\mathbf{i}$ the quantum torus $*$-algebra $\mathrm{T}_{\mathbf{i}}^{q}$, and to a mutation $\mathbf{i} \longrightarrow \mathbf{i}^{\prime}$ a map of the fraction fields $\operatorname{Frac}\left(\mathrm{T}_{\mathbf{i}^{\prime}}^{q}\right) \longrightarrow \operatorname{Frac}\left(\mathrm{T}_{\mathbf{i}}^{q}\right)$, given by a $q$-deformation of formulas (3) $)^{2}:$ Set $q_{k}:=q^{1 / h_{k}}$,

$$
\left(\mu_{k}^{q}\right)^{*}\left(X_{i}\right):= \begin{cases}X_{k}^{-1} & \text { if } i=k, \\ X_{i}\left(\prod_{a=1}^{\left|\varepsilon_{i k}\right|}\left(1+q_{k}^{2 a-1} X_{k}^{-\operatorname{sgn}\left(\varepsilon_{\mathrm{ik}}\right)}\right)\right)^{-\varepsilon_{i k}} & \text { if } i \neq k .\end{cases}
$$

One uses Theorem 7.2 in loc. cit. to prove that $\eta^{q}$ sends trivial seed cluster transformations to the identity maps.

The chiral dual to a seed $\mathbf{i}=(I, \varepsilon, d)$ is a seed $\mathbf{i}^{o}:=(I,-\varepsilon, d)$. Mutations commute with the chiral duality on seeds. Therefore a cluster $\mathcal{X}$-variety $\mathcal{X}$ (respectively $\mathcal{A}$-variety $\mathcal{A}$ ), gives rise to the chiral dual cluster $\mathcal{X}$-variety (respectively $\mathcal{A}$-variety) denoted by $\mathcal{X}^{o}$ (respectively $\mathcal{A}^{0}$ ). They are related, see Lemmas 2.1-2.2.

The Langlands dual to a seed $\mathbf{i}=(I, \varepsilon, d)$ is the seed $\mathbf{i}^{\vee}=\left(I, \varepsilon^{\vee}, d^{\vee}\right)$, where $d_{i}^{\vee}:=d_{i}^{-1}$ and

$$
\varepsilon_{i j}^{\vee}=-\varepsilon_{j i}^{\vee}:=\widehat{d}_{i}^{-1} \varepsilon_{i j} \widehat{d}_{j}, \quad \widehat{d}_{i}:=d_{i}^{-1} .
$$

The Langlands duality on seeds commutes with mutations. Therefore it gives rise to the Langlands dual cluster $\mathcal{A}$-, and $\mathcal{X}$-varieties, denoted $\mathcal{A}^{\vee}$ and $\mathcal{X}^{\vee}$.

Below we skip the subscript $|\mathbf{i}|$ encoding the corresponding cluster ensemble whenever possible.

\subsection{Connections between quantum $\mathcal{X}$-varieties}

There are three ways to alter the space $\mathcal{X}_{|\mathbf{i}|, q}$ :

(i) change $q$ to $q^{-1}$,

(ii) change $\mathbf{i}$ to $\mathbf{i}^{o}$,

\footnotetext{
${ }^{2}$ See also a more transparent and less computational definition given later on in Section 3 of [FG3].
} 
(iii) change the quantum space $\mathcal{X}_{|\mathbf{i}|, q}$ to the opposite quantum space $\mathcal{X}_{|\mathbf{i}|, q}^{\text {opp }}$.

(In (iii) we change every quantum torus from which we glue the space to the opposite one).

The following lemma tells that the resulting three quantum spaces are canonically isomorphic:

Lemma 2.1 a) There is a canonical isomorphism of quantum spaces

$$
\alpha_{\mathcal{X}}^{q}: \mathcal{X}_{|\mathbf{i}|, q} \longrightarrow \mathcal{X}_{|\mathbf{i}|, q^{-1}}^{\text {opp }}, \quad\left(\alpha_{\mathcal{X}}^{q}\right)^{*}: X_{i} \longmapsto X_{i} .
$$

(Given in on the generators of any cluster coordinate system by $X_{i} \longmapsto X_{i}$ ).

b) There is a canonical isomorphism of quantum spaces

$$
i_{\mathcal{X}}^{q}: \mathcal{X}_{|\mathbf{i}|, q} \longrightarrow \mathcal{X}_{\left|\mathbf{i}^{\circ}\right|, q^{-1}}, \quad\left(i_{\mathcal{X}}^{q}\right)^{*}: X_{i}^{o} \longmapsto X_{i}^{-1}
$$

(Given in any cluster coordinate system by $X_{i}^{o} \longmapsto X_{i}^{-1}$, where $X_{i}^{o}$ are the generators of $\mathcal{X}_{\mathbf{i}^{o}, q^{-1}}$ ).

c) There is a canonical isomorphism of quantum spaces

$$
\beta_{\mathcal{X}}^{q}:=\alpha_{\mathcal{X}}^{q} \circ i_{\mathcal{X}}^{q}: \mathcal{X}_{|\mathbf{i} \mathbf{\circ}|, q} \longrightarrow \mathcal{X}_{|\mathbf{i}|, q}^{\text {opp }}, \quad X_{i} \longmapsto X_{i}^{o-1} .
$$

Proof. Apparently each of the three maps is an isomorphism of the corresponding seed quantum tori algebras. For example, in the case b) we have

$$
i_{\mathcal{X}}^{q}\left(\left(q^{-1}\right)^{-\widehat{\varepsilon}_{i j}^{o}} X_{i}^{o} X_{j}^{o}\right)=q^{-\widehat{\varepsilon}_{i j}} X_{i}^{-1} X_{j}^{-1} .
$$

So we need to check that they commute with the mutations.

a) Let us assume first that $\varepsilon_{i k}=a<0$. The claim results from the fact that the following two compositions are equal (observe that $\alpha^{*}$ is an antiautomorphism):

$$
\begin{gathered}
X_{i} \stackrel{\mu_{k}^{*}}{\longmapsto} X_{i} \prod_{b=1}^{a}\left(1+q_{k}^{2 b-1} X_{k}\right) \stackrel{\alpha^{*}}{\longmapsto} \prod_{b=1}^{a}\left(1+q_{k}^{2 b-1} X_{k}\right) X_{i} ; \\
X_{i} \stackrel{\alpha^{*}}{\longmapsto} X_{i} \stackrel{\left(\mu_{k}^{o}\right)^{*}}{\longmapsto} X_{i} \prod_{b=1}^{a}\left(1+q_{k}^{-(2 b-1)} X_{k}\right) .
\end{gathered}
$$

The computation in the case $\varepsilon_{i k}>0$ is similar.

b) To check that $i_{\mathcal{X}}^{q} \circ \mu_{k}^{*}=\mu_{k}^{*} \circ i_{\mathcal{X}}^{q}$ we calculate each of the maps on the generator $X_{i}$. Let us assume $\varepsilon_{i k}=-a<0$. Then $\varepsilon_{i k}^{o}=a$, and one has

$$
\begin{gathered}
i_{\mathcal{X}}^{q} \circ \mu_{k}^{*}\left(X_{i}^{o \prime}\right)=X_{i}^{-1} \prod_{b=1}^{a}\left(1+q^{2 b-1} X_{k}^{-1}\right), \\
\mu_{k}^{*} \circ i_{\mathcal{X}}^{q}\left(X_{i}^{o \prime}\right)=\left(X_{i} \prod_{b=1}^{a}\left(1+q^{-(2 b-1)} X_{k}^{-1}\right)^{-1}\right)^{-1}=\prod_{b=1}^{a}\left(1+q^{-(2 b-1)} X_{k}^{-1}\right) X_{i}^{-1}=X_{i}^{-1} \prod_{b=1}^{a}\left(1+q^{2 b-1} X_{k}^{-1}\right) .
\end{gathered}
$$

The case $\varepsilon_{i k}>0$ is similar. One can deduce it to the case $\varepsilon_{i k}<0$ since $\mu_{k} \circ \mu_{k}=\mathrm{Id}$, and $\varepsilon_{i k}^{\prime}=-\varepsilon_{i k}$. The part b) is proved.

c) Follows from a) and b). The lemma is proved. 
Lemma 2.2 The cluster ensembles related to the seeds $\mathbf{i}$ and $\mathbf{i}^{\mathbf{o}}$ are canonically isomorphic as pairs of varieties. The isomorphism is provided by the following maps:

$$
\operatorname{Id}: \mathcal{A}_{|\mathbf{i}|} \longrightarrow \mathcal{A}_{\mathbf{i}^{\mathbf{o}}} ; \quad i_{\mathcal{X}}: \mathcal{X}_{|\mathbf{i}|} \longrightarrow \mathcal{X}_{\mid \mathbf{i} \mathbf{o}} \text {. }
$$

Proof. In a given cluster coordinate system our maps are obviously isomorphisms. The compatibility with $\mathcal{X}$-cluster transformations is the part b) of Lemma 2.1; for the $\mathcal{A}$-cluster transformations we have

$$
A_{k}^{o} A^{o \prime}{ }_{k}=\prod_{\varepsilon_{k i}^{o}>0}\left(A_{i}^{o}\right)^{\varepsilon_{k i}^{o}}+\prod_{\varepsilon_{k i}^{o}<0}\left(A_{i}^{o}\right)^{-\varepsilon_{k i}^{o}}=\prod_{\varepsilon_{k i}<0} A_{i}^{-\varepsilon_{k i}}+\prod_{\varepsilon_{k i}>0} A_{i}^{\varepsilon_{k i}}=A_{k} A_{k}^{\prime}
$$

Compatibility with the projection $p$ is clear. The lemma is proved.

\section{Motivation: *-quantization of cluster $\mathcal{X}$-varieties}

\section{$3.1 *$-quantization of the space $\mathcal{X}^{+}$via the quantum logarithm}

Let $\left\{X_{i}\right\}$ be coordinates on the cluster $\mathcal{X}$-variety corresponding to a seed i. Since by definition the functions $X_{i}$ are strictly positive at the points of $\mathcal{X}^{+}$, we can introduce the logarithmic coordinates $x_{i}:=\log X_{i}$ on $\mathcal{X}^{+}$. For every seed $\mathbf{i}$ they provide an isomorphism

$$
\beta_{\mathbf{i}}: \mathcal{X}_{\mathbf{i}}^{+} \stackrel{\sim}{\longrightarrow} \mathbb{R}^{I} ; \quad t \longmapsto\left\{x_{i}(t)\right\} .
$$

For a mutation $\mu_{k}: \mathbf{i} \rightarrow \mathbf{i}^{\prime}$ there is a gluing map

$$
\beta_{\mathbf{i} \rightarrow \mathbf{i}^{\prime}}: \mathcal{X}_{\mathbf{i}}^{+} \longrightarrow \mathcal{X}_{\mathbf{i}^{\prime}}^{+}, \quad \beta_{\mathbf{i} \rightarrow \mathbf{i}^{\prime}}\left(x_{i}^{\prime}\right)= \begin{cases}x_{i}-\varepsilon_{i k} \log \left(1+e^{-\operatorname{sgn}\left(\varepsilon_{i k}\right) x_{k}}\right) & i \neq k, \\ -x_{k} & i=k\end{cases}
$$

To prepare the soil for quantization, let us look at this from a different point of view. Let $\mathrm{Com}^{*}$ be the category of commutative topological $*$-algebras over $\mathbb{C}$. Recall the cluster modular groupoid $\mathcal{G}_{|\mathbf{i}|}$. There is a contravariant functor

$$
\beta: \mathcal{G}_{|\mathbf{i}|} \longrightarrow \mathrm{Com}^{*} \text {. }
$$

Namely, we assign to a seed $\mathbf{i}$ a commutative topological *-algebra $S\left(\mathcal{X}_{\mathbf{i}}^{+}\right)$of smooth complex valued functions in $\mathcal{X}_{\mathbf{i}}^{+}$with $* f:=\bar{f}$, and to a mutation $\mathbf{i} \rightarrow \mathbf{i}^{\prime}$ a homomorphism $\beta_{\mathbf{i} \rightarrow \mathbf{i}^{\prime}}^{*}: S\left(\mathcal{X}_{\mathbf{i}^{\prime}}^{+}\right) \longrightarrow S\left(\mathcal{X}_{\mathbf{i}}^{+}\right)$.

Let $\mathcal{C}$ be a category whose morphisms are $\mathbb{C}$-vector spaces. Projectivisation $P \mathcal{C}$ of the category $\mathcal{C}$ as a new category with the same objects as $\mathcal{C}$, and morphisms given by $\operatorname{Hom}_{P \mathcal{C}}\left(C_{1}, C_{2}\right):=$ $\operatorname{Hom}_{\mathcal{C}}\left(C_{1}, C_{2}\right) / U(1)$, where $U(1)$ is the multiplicative group of complex numbers with absolute value 1 . A projective functor $F: \mathcal{G} \rightarrow \mathcal{C}$ is a functor from $\mathcal{G}$ to $P \mathcal{C}$.

Let $\mathcal{C}^{*}$ be the category of topological $*$-algebras. Two functors $F_{1}, F_{2}: \mathcal{C} \longrightarrow \mathcal{C}^{*}$ essentially coincide if there exists a third functor $F$ and natural transformations $F_{1} \rightarrow F, F_{2} \rightarrow F$ providing for every object $C$ dense inclusions $F_{1}(C) \hookrightarrow F_{3}(C), F_{1}(C) \hookrightarrow F_{3}(C)$.

Definition 3.1 A quantization of the space $\mathcal{X}_{|\mathbf{i}|}^{+}$is a family of contravariant projective functors

$$
\kappa_{|\mathbf{i}|}^{\hbar}: \mathcal{G}_{|\mathbf{i}|} \longrightarrow \mathcal{C}^{*}
$$

depending smoothly on a real parameter $\hbar$, related to the original Poisson manifold $\mathcal{X}_{|\mathbf{i}|}^{+}$as follows:

i) The limit $\kappa_{|\mathbf{i}|}:=\lim _{\hbar \rightarrow 0} \kappa_{|\mathbf{i}|}^{\hbar}$ exists and essentially coincides with the functor $\beta$ defining $\mathcal{X}_{|\mathbf{i}|}^{+}$.

ii) The Poisson bracket given by $\lim _{\hbar \rightarrow 0}\left[f_{1}, f_{2}\right] / \hbar$ is defined and coincides with the one on $\mathcal{X}_{|\mathbf{i}|}^{+}$. 
Let us define a quantization functor $\kappa^{\hbar}=\kappa_{|\mathbf{i}|}^{\hbar}$. We assign to every seed $\mathbf{i}$ the Heisenberg $*$-algebra $\mathcal{H}_{\mathrm{i}}^{\hbar}$. It is a topological $*$-algebra over $\mathbb{C}$ generated by the elements $x_{i}$ such that

$$
\left[x_{j}, x_{k}\right]=2 \pi i \hbar \widehat{\varepsilon}_{j k} ; \quad x_{j}^{*}=x_{j} ; \quad q=e^{\pi i \hbar} .
$$

Further, let us assign to mutation $\mu_{k}: \mathbf{i} \rightarrow \mathbf{i}^{\prime}$ a homomorphism of topological $*$-algebras

$$
\kappa^{\hbar}\left(\mu_{k}\right): \mathcal{H}_{\mathbf{i}^{\prime}}^{\hbar} \longrightarrow \mathcal{H}_{\mathbf{i}}^{\hbar}
$$

We employ the quantum logarithm $\phi^{\hbar}(z)$, see (22). Denote by $x_{i}^{\prime}$ the generators of $\mathcal{H}_{\mathbf{i}^{\prime}}^{\hbar}$. Set

$$
\hbar_{k}:=\widehat{d}_{k} \hbar ; \quad \kappa^{\hbar}\left(\mu_{k}\right): x_{i}^{\prime} \longmapsto \begin{cases}x_{i}-\varepsilon_{i k} \phi^{\hbar_{k}}\left(-\operatorname{sgn}\left(\varepsilon_{i k}\right) x_{k}\right) & \text { if } k \neq i, \\ -x_{i} & \text { if } k=i .\end{cases}
$$

Claim 3.2 a) Formulas (8) provide a morphism of $*$-algebras $\kappa_{|\mathbf{i}|}^{\hbar}\left(\mu_{k}\right): \mathcal{H}_{\mathbf{i}^{\prime}}^{\hbar} \longrightarrow \mathcal{H}_{\mathbf{i}}^{\hbar}$.

b) The collection of $*$-algebras $\left\{\mathcal{H}_{\mathbf{i}}\right\}$ and morphisms $\left\{\kappa_{|\mathbf{i}|}^{\hbar}\left(\mu_{k}\right)\right\}$ provide a quantization functor

$$
\kappa_{|\mathbf{i}|}^{\hbar}: \mathcal{G}_{|\mathbf{i}|} \longrightarrow P \mathcal{C}^{*}
$$

c) Let $\hbar^{\vee}:=1 / \hbar$. Then there are isomorphisms

$$
\mathcal{H}_{\mathrm{i}}^{\hbar} \longrightarrow \mathcal{H}_{\mathrm{i}^{\vee}}^{\hbar^{\vee}}, \quad x_{i} \longmapsto x_{i}^{\vee}:=\frac{x_{i}}{\widehat{d}_{i} \hbar} ;
$$

They give rise to a natural transformation of functors $\kappa_{|\mathbf{i}|}^{\hbar} \longrightarrow \kappa_{\mid \mathbf{i} \vee}^{\hbar^{\vee}}$.

Justification. a) Property A3 of the function $\phi^{\hbar}(x)$, see Section 4, guarantees that the morphism $\kappa^{\hbar}\left(\mu_{k}\right)$ preserves the real structure. It follows from Property A1 that when $\hbar \rightarrow 0$ the limit of the quantum formula (8) exists and coincides with the mutation formula (6) for the logarithmic coordinates $x_{i}$.

b) To check that we have a functor one needs to check first that mutation formulas are compatible with the transformations $\kappa^{\hbar}\left(\mu_{k}\right)$. This is a straitforward calculation using Property A5. Then one has to check that the defining relations for the groupoid $\mathcal{G}_{|\mathbf{i}|}$ are mapped to zero. Here we need the results of Sections 3.2-3.3 of [FG2] and the following well known lemma:

Lemma 3.3 Suppose that $A, B$ are selfadjoint operators, $[A, B]=-\lambda$ is a scalar, and $f(z)$ is a continuous function with primitive $F(z)$. Then

$$
e^{A+f(B)}=e^{A} \exp \left\{\frac{1}{\lambda} \int_{B}^{B+\lambda} f(z) d z\right\}:=e^{A} \exp \left(\frac{F(B+\lambda)-F(B)}{\lambda}\right) .
$$

c) Thanks to formula (5) the map $x_{i} \longmapsto x_{i}^{\vee}$ is an $*$-algebra homomorphism:

$$
\left[x_{i}^{\vee}, x_{j}^{\vee}\right]=\frac{\left[x_{i}, x_{j}\right]}{\widehat{d}_{i} \widehat{d}_{j} \hbar^{2}}=2 \pi i \hbar^{\vee} \widehat{\varepsilon}_{i j} / \widehat{d}_{i} \widehat{d}_{j} \stackrel{5}{=} 2 \pi i \hbar^{\vee} \widehat{\varepsilon}_{i j}^{\vee}
$$

To verify that it commutes with mutation homomorphisms we use Properties A2 and A4 of the function $\phi^{\hbar}(x)$, observing that

$$
\frac{x_{i}+\left|\varepsilon_{i k}\right| \phi^{\hbar_{k}}\left(x_{k}\right)}{\widehat{d}_{i} \hbar}=\frac{x_{i}}{\widehat{d}_{i} \hbar}+\frac{\left|\widehat{d}_{i}^{-1} \varepsilon_{i k} \widehat{d}_{k}\right| \phi^{\widehat{d}_{k} \hbar}\left(x_{k}\right)}{\widehat{d}_{k} \hbar} \stackrel{5}{=}+A 4 \quad x_{i}^{\vee}+\left|\varepsilon_{i k}^{\vee}\right| \phi^{\hbar_{k}^{\vee}}\left(x_{k}^{\vee}\right)
$$




\section{$3.2 \quad$ Modular double of a cluster $\mathcal{X}$-variety and $*$-quantization of the space $\mathcal{X}^{+}$} Set

$$
q:=e^{\pi i \hbar}, \quad q^{\vee}:=e^{\pi i / \hbar}, \quad \hbar \in \mathbb{R} .
$$

Definition 3.4 The modular double $\mathcal{X}_{|\mathbf{i}|, q} \times \mathcal{X}_{|\mathbf{i} \vee|, q}$ of a quantum cluster $\mathcal{X}_{\text {-variety }} \mathcal{X}_{|\mathbf{i}|, q}$ is a contravariant functor

$$
\eta_{|\mathbf{i}|}^{q} \otimes \eta_{\left|\mathbf{i}^{\vee}\right|}^{q^{\vee}}: \mathcal{G}_{|\mathbf{i}|} \longrightarrow \text { QTor* }
$$

So we assign to a seed $\mathbf{i}$ a quantum torus algebra $\mathrm{T}_{\mathbf{i}}^{q} \otimes \mathrm{T}_{\mathbf{i}^{\vee}}^{q^{\vee}}$, and to a mutation $\mu_{k}: \mathbf{i} \rightarrow \mathbf{i}^{\prime}$ a positive $*$-homomorphism of the fraction fields of the quantum torus algebras

$$
\eta_{|\mathbf{i}|}^{q}\left(\mu_{k}\right) \otimes \eta_{|\mathbf{i} \vee|}^{q^{\vee}}\left(\mu_{k}\right): \mathbb{T}_{\mathbf{i}^{\prime}}^{q} \otimes \mathbb{T}_{\mathbf{i}^{\vee \vee}}^{q^{\vee}} \longrightarrow \mathbb{T}_{\mathbf{i}}^{q} \otimes \mathbb{T}_{\mathbf{i}^{\vee}}^{q^{\vee}}, \quad \mathbb{T}:=\operatorname{Frac}(\mathrm{T})
$$

We want to relate the modular double $\mathcal{X}_{|\mathbf{i}|, q} \times \mathcal{X}_{|\mathbf{i} \vee|, q^{\vee}}$ with the quantization of the space $\mathcal{X}_{|\mathbf{i}|}^{+}$. We are going to define a natural transformation of functors $\eta_{|\mathbf{i}|}^{q} \otimes \eta_{\mathcal{E} \vee}^{q^{\vee}} \longrightarrow \kappa_{|\mathbf{i}|}^{\hbar}$.

We use the following easy fact. Assume that $\left[y_{i}, y_{j}\right]$ is a scalar. Then we have

$$
e^{y_{i}} e^{y_{j}}=e^{\left[y_{i}, y_{j}\right]} e^{y_{j}} e^{y_{i}}
$$

Let $\mathbf{i}$ be a seed. Denote by $X_{i}$ the generators of $\mathbb{T}_{\mathbf{i}}^{q}$, and by $X_{i}^{\vee}$ the generators of $\mathbb{T}_{\mathbf{i}^{\vee}}^{{ }^{\vee}}$. It is easy to check using (10) that there are the following homomorphisms:

$$
l_{\mathbf{i}}: \mathbb{T}_{\mathbf{i}}^{q} \longrightarrow \mathcal{H}_{\mathbf{i}}^{h}, \quad X_{i} \longmapsto e^{x_{i}}, \quad \text { and } \quad l_{\mathbf{i}}^{\vee}: \mathbb{T}_{\mathbf{i} \vee}^{q^{\vee}} \longrightarrow \mathcal{H}_{\mathbf{i}}^{\hbar}, \quad X_{i}^{\vee} \longmapsto e^{x_{i}^{\vee}}
$$

They evidently commute with the $*$-structures. Their images commute. Indeed, since $\widehat{\varepsilon}_{i j} \in \mathbb{Z}$ one has $e^{\left[x_{i}, x_{j} / \hbar\right]}=e^{2 \pi i \widehat{\varepsilon}_{i j}}=1$. So $e^{x_{i}}$ commutes with $e^{x_{j} / \hbar}$. Therefore they give rise to a homomorphism of the tensor product:

$$
L_{\mathbf{i}}:=l_{\mathbf{i}} \otimes l_{\mathbf{i}}^{\vee}: \mathbb{T}_{\mathbf{i}}^{q} \otimes \mathbb{T}_{\mathbf{i}^{\vee}}^{q^{\vee}} \longrightarrow \mathcal{H}_{\mathbf{i}}^{\hbar}
$$

Proposition 3.5 For any mutation $\mu_{k}: \mathbf{i} \rightarrow \mathbf{i}^{\prime}$ the following diagram, where the left vertical arrow is the map $\eta^{q}\left(\mu_{k}\right) \otimes \eta^{q^{\vee}}\left(\mu_{k}\right)$, and the right one is $\kappa^{\hbar}\left(\mu_{k}\right)$, is commutative:

$$
\begin{array}{crc}
\mathbb{T}_{\mathbf{i}}^{q} \otimes \mathbb{T}_{\mathbf{i}}^{q^{\vee}} & \stackrel{L_{\mathbf{i}}}{\longrightarrow} & \mathcal{H}_{\mathbf{i}}^{\hbar} \\
\eta^{q, q^{\vee} \uparrow} & \uparrow \kappa^{\hbar} \\
\mathbb{T}_{\mathbf{i}^{\prime}}^{q} \otimes \mathbb{T}_{\mathbf{i}^{\prime}}^{q^{\vee}} & \stackrel{L_{\mathbf{i}^{\prime}}}{\longrightarrow} & \mathcal{H}_{\mathbf{i}^{\prime}}^{\hbar}
\end{array}
$$

Proof. We need Lemma 3.3. The case $i=k$ is trivial, so we assume that $i \neq k$. Let $\varepsilon_{i k}=-a \leq 0$. Then applying the lemma we get

$$
\kappa_{|\mathbf{i}|}^{\hbar}\left(\mu_{k}\right) L_{\mathbf{i}^{\prime}}\left(X_{i}^{\prime} \otimes 1\right)=\kappa^{\hbar}\left(\mu_{k}\right) e^{x_{i}^{\vee}}=e^{x_{i}+a \phi^{\hbar} k\left(x_{k}\right)}=\frac{e^{x_{i}}}{2 \pi i a \hbar_{k}} \exp \left(\int_{x_{k}}^{x_{k}+2 \pi i \hbar_{k} a} a \phi^{\hbar_{k}}(z) d z\right)=
$$




$$
\begin{gathered}
\frac{e^{x_{i}}}{2 \pi i \hbar_{k}} \exp \left(\int_{-\infty}^{x_{k}}\left(\phi^{\hbar_{k}}\left(z+2 \pi i \hbar_{k} a\right)-\phi^{\hbar_{k}}(z)\right) d z\right) \stackrel{A 5}{=} \frac{e^{x_{i}}}{2 \pi i \hbar_{k}} \exp \left(\int_{-\infty}^{x_{k}} \sum_{b=1}^{a} \frac{2 \pi i \hbar_{k}}{\left.e^{-z-i \pi(2 b-1) \hbar_{k}+1} d z\right)=}\right. \\
e^{x_{i}} \prod_{b=1}^{a}\left(1+q_{k}^{2 a-1} e^{x_{k}}\right)=L_{\mathbf{i}}\left(X_{i} \prod_{b=1}^{a}\left(1+q_{k}^{2 a-1} X_{k}\right)\right)=L_{\mathbf{i}}\left(\eta_{|\mathbf{i}|}^{q}\left(\mu_{k}\right) \otimes \eta_{|\mathbf{i} \vee|}^{q^{\vee}}\left(\mu_{k}\right)\right)\left(X_{i}^{\prime} \otimes 1\right) .
\end{gathered}
$$

The calculation in the case $\varepsilon_{i k}=a \geq 0$ is similar. The proposition is proved.

Claim 3.6 The collection of homomorphisms $\left\{L_{\mathbf{i}}\right\}$ provides a morphism of functors

$$
\mathbb{L}^{\hbar}: \eta_{|\mathbf{i}|}^{q} \otimes \eta_{|\mathbf{i} \vee|}^{q^{\vee}} \longrightarrow \kappa_{|\mathbf{i}|}^{\hbar}
$$

Justification. Is given by Proposition 3.5.

Representations of the quantized $\mathcal{X}_{|\mathbf{i}|}^{+}$-space. The following definition serves as a motivation of the construction of intertwiners presented below.

Definition 3.7 A projective $*$-representation of the quantized $\mathcal{X}_{|\mathbf{i}|}^{+}$-space is the following data:

i) A projective functor

$$
\mathcal{L}_{|\mathbf{i}|}: \mathcal{G}_{|\mathbf{i}|} \longrightarrow \text { the category of Hilbert spaces. }
$$

It includes for each object $\mathbf{i}$ of $\mathcal{G}_{|\mathbf{i}|}$ a Hilbert spaces $\mathbb{L}_{\mathbf{i}}$, and for every mutation $\mu_{k}: \mathbf{i} \rightarrow \mathbf{i}^{\prime}$ a unitary operator, defined up to a scalar of absolute value 1 :

$$
\mathbf{K}_{\mathbf{i}, \mathbf{i}^{\prime}}: \mathbb{L}_{\mathbf{i}} \longrightarrow \mathbb{L}_{\mathbf{i}^{\prime}}
$$

ii) A *-representation $\rho_{\mathbf{i}}$ of the Heisenberg algebra $\mathcal{H}_{\mathbf{i}}^{\hbar}$ in the Hilbert space $\mathbb{L}_{\mathbf{i}}$.

iii) The operators $\mathbf{K}_{\mathbf{i}, \mathbf{i}^{\prime}}$ intertwine the representations $\rho_{\mathbf{i}}$ and $\rho_{\mathbf{i}^{\prime}}$ :

$$
\rho_{\mathbf{i}}(s)=\mathbf{K}_{\mathbf{i}, \mathbf{i}^{\prime}}^{-1} \rho_{\mathbf{i}^{\prime}}\left(\kappa^{\hbar}\left(\mu_{k}\right)(s)\right) \mathbf{K}_{\mathbf{i}, \mathbf{i}^{\prime}}, \quad s \in \mathcal{H}_{\mathbf{i}}^{\hbar}
$$

The morphisms of the representations of the quantum $\mathcal{X}_{|\mathbf{i}|}^{+}$-space are defined in an obvious way.

Representations of the mapping class group $\Gamma_{|\mathbf{i}|}$. Restricting the functor $\rho_{|\mathbf{i}|}$ to the group of automorphisms of an object of the groupoid $\mathcal{G}_{|\mathbf{i}|}$ we get a projective unitary representation of $\Gamma_{|\mathbf{i}|}$.

The Heisenberg algebra $\mathcal{H}_{\mathbf{i}}^{\hbar}$ has a family of irreducible $*$-representation by operators in a Hilbert space. These representations are characterized by the central character $\chi$.

The collection of the Hilbert spaces $\left\{\mathbb{L}_{\mathbf{i}}\right\}$ and representations $\left\{\rho_{\mathbf{i}}\right\}$ is by no means canonical: it depends, for example, on the choice of polarization of the Heisenberg algebra. Once choosen, it determines the intertwiners $\mathbf{K}_{\mathbf{i}, \mathbf{i}^{\prime}}$. Below we introduce a canonical representation of the chiral double of the quantized space $\mathcal{X}_{|\mathbf{i}|}^{+}$, defined by using the Hilbert spaces $L^{2}\left(\mathcal{A}_{\mathbf{i}}^{+}\right)$. 


\section{The intertwiner}

\subsection{A bimodule structure on functions on the $\mathcal{A}$-space}

Let $X$ be an algebra. Recall that $M$ is a bimodule over $X$ if $X$ acts on $M$ from the left as well as from the right, and these two actions commute. So $M$ is an $X \otimes X^{\mathrm{opp}}$-module, where $X^{\mathrm{opp}}$ is the algebra with the product $x * y:=y x$.

Let us choose a seed i. Recall the algebra $\mathbb{Q}\left[\mathcal{A}_{\mathbf{i}}\right]$ of regular functions on the seed torus $\mathcal{A}_{\mathbf{i}}$. We assume that $q \in \mathbb{C}^{*}$. For each $i \in I$ let us define commuting algebra homomorphisms

$$
t_{i}^{ \pm}: \mathbb{Q}\left[\mathcal{A}_{\mathbf{i}}\right] \longrightarrow \mathbb{Q}\left[\mathcal{A}_{\mathbf{i}}\right] ; \quad t_{i}^{ \pm}:\left\{\begin{array}{l}
A_{i} \longmapsto q^{ \pm \widehat{d}_{i}} A_{i} \\
A_{j} \longmapsto A_{j}
\end{array} \quad j \neq i .\right.
$$

Since $\varepsilon_{i i}=0, A_{i}$ does not appear in the monomial $p^{*} X_{i}$, and so the operator of multiplication by $p^{*} X_{i}$ commutes with $t_{i}^{ \pm}$. Let us define an $T_{\mathbf{i}}^{q}$-bimodule structure on $\mathbb{Q}\left[\mathcal{A}_{\mathbf{i}}\right]$. The left and right actions of the generator $X_{i}$ on $f \in \mathbb{Q}\left[\mathcal{A}_{\mathbf{i}}\right]$ are given by

$$
X_{i} \circ f:=p^{*} X_{i} \cdot t_{i}^{-}(f), \quad f \circ X_{i}:=p^{*} X_{i} \cdot t_{i}^{+}(f),
$$

Lemma 4.1 The operators (12) provide $\mathbb{Q}\left[\mathcal{A}_{\mathbf{i}}\right]$ with a structure of a bimodule over the algebra $T_{\mathbf{i}}^{q}$.

Proof. Observe that one has

$$
t_{j}^{+}\left(p^{*} X_{i}\right) \cdot p^{*} X_{j}=t_{i}^{-}\left(p^{*} X_{j}\right) \cdot p^{*} X_{i}=q^{\widehat{\varepsilon}_{i j}} p^{*} X_{i} p^{*} X_{j} .
$$

Indeed, the first term equals to $q^{\varepsilon_{i j} d_{j}} p^{*} X_{i} \cdot p^{*} X_{j}$, and the second is $q^{-\varepsilon_{j i} d_{i}} p^{*} X_{i} \cdot p^{*} X_{j}$. One has

$$
\begin{aligned}
& q^{-\widehat{\varepsilon}_{i j}} X_{i} X_{j} \circ f=q^{-\widehat{\varepsilon}_{i j}} p^{*} X_{i} \cdot t_{i}^{-}\left(p^{*} X_{j}\right) \cdot t_{i}^{-} t_{j}^{-}(f)=p^{*} X_{i} \cdot p^{*} X_{j} \cdot t_{i}^{-} t_{j}^{-}(f) . \\
& f \circ q^{-\widehat{\varepsilon}_{i j}} X_{i} X_{j}=q^{-\widehat{\varepsilon}_{i j}} p^{*} X_{j} \cdot t_{j}^{+}\left(p^{*} X_{i}\right) \cdot t_{j}^{+} t_{i}^{+}(f)=p^{*} X_{i} \cdot p^{*} X_{j} \cdot t_{i}^{+} t_{j}^{+}(f) .
\end{aligned}
$$

Since the right hand sides are evidently symmetric in $i, j$ we have the desired relations, and hence the left and right actions of the quantum algebra torus. Further, the two actions commute:

$$
\begin{gathered}
X_{i} \circ\left(f \circ X_{j}\right)=X_{i} \circ\left(t_{j}^{+}(f) p^{*} X_{j}\right)=p^{*} X_{i} t_{i}^{-}\left(p^{*} X_{j}\right) t_{i}^{-} t_{j}^{+}(f) . \\
\left(X_{i} \circ f\right) \circ X_{j}=\left(p^{*} X_{i} t_{i}^{-}(f)\right) \circ X_{j}=p^{*} X_{j} t_{j}^{+}\left(p^{*} X_{i}\right) t_{j}^{+} t_{i}^{-}(f) .
\end{gathered}
$$

The lemma is proved.

The logarithmic version of the bimodule structure. Since the coordinate functions $A_{i}$ are positive on the space $\mathcal{A}^{+}$, one can introduce new coordinates $a_{j}:=\log A_{j}$. They provide an isomorphism $\alpha_{\mathbf{i}}: \mathcal{A}_{\mathbf{i}}^{+} \stackrel{\sim}{\longrightarrow} \mathbb{R}^{I}$. Set $d a:=d a_{1} \wedge \cdots \wedge d a_{|I|}$. There is a Hilbert space $L^{2}\left(\mathcal{A}_{\mathbf{i}}^{+}\right)$with a scalar product

$$
(f, g):=\int_{\mathcal{A}_{\mathbf{i}}^{+}} f(a) \overline{g(a)} d a .
$$

Apparently the form $d a$ changes the sign under a mutation $\mathbf{i} \rightarrow \mathbf{i}^{\prime}$. So the Hilbert spaces $L^{2}\left(\mathcal{A}_{\mathbf{i}}^{+}\right)$ for different seeds $\mathbf{i}$ are naturally identified. Consider the following operators in $L^{2}\left(\mathcal{A}_{\mathbf{i}}^{+}\right)$:

$$
\widehat{x}_{j}^{-}:=-\pi i \hbar \widehat{d}_{j} \frac{\partial}{\partial a_{j}}+\sum_{k} \varepsilon_{j k} a_{k}, \quad \widehat{x}_{j}^{+}:=\pi i \hbar \widehat{d}_{j} \frac{\partial}{\partial a_{j}}+\sum_{k} \varepsilon_{j k} a_{k} .
$$


Lemma 4.2 The operators $\left\{\widehat{x}_{j}^{ \pm}\right\}$provide the Hilbert space $L^{2}\left(\mathcal{A}_{\mathbf{i}}^{+}\right)$with a structure of a bimodule over the *-algebra $\mathcal{H}_{\mathrm{i}}^{\hbar}$.

Proof. These operators are selfadjoint and one has

$$
\left[\widehat{x}_{j}^{-}, \widehat{x}_{k}^{-}\right]=2 \pi i \hbar \widehat{\varepsilon}_{j k} ; \quad\left[\widehat{x}_{j}^{+}, \widehat{x}_{k}^{+}\right]=-2 \pi i \hbar \widehat{\varepsilon}_{j k}, \quad\left[\widehat{x}_{j}^{-}, \widehat{x}_{k}^{+}\right]=0 \quad \text { for any } j, k \in I .
$$

The lemma is proved.

Remark. There is an automorphism $x_{j} \longmapsto-x_{j}$ of the Heisenberg algebra $\mathcal{H}_{\mathbf{i}}$. Similarly there is an automorphism $X_{j} \longmapsto X_{j}^{-1}$ of the quantum torus algebra $T_{\mathbf{i}}^{q}$.

\subsection{The intertwiner via the quantum dilogarithm}

Let $\mu_{k}: \mathbf{i} \rightarrow \mathbf{i}^{\prime}$ be a mutation. By Lemma 4.2 for each seed $\mathbf{i}$ the Hilbert space $L^{2}\left(\mathcal{A}_{\mathbf{i}}^{+}\right)$has a natural $\mathcal{H}_{\mathbf{i}}^{\hbar}$-bimodule structure. According to Lemma 2.1, this is the same as the $\mathcal{H}_{\mathbf{i}}^{h} \otimes \mathcal{H}_{\mathbf{i}^{\circ}}^{\hbar}$-module structure. Our goal is to define an operator

$$
\mathbf{K}_{\mathbf{i} \rightarrow \mathbf{i}^{\prime}}: L^{2}\left(\mathcal{A}_{\mathbf{i}}^{+}\right) \longrightarrow L^{2}\left(\mathcal{A}_{\mathbf{i}^{\prime}}^{+}\right)
$$

intertwining the $\mathcal{H}_{\mathbf{i}}^{\hbar} \otimes \mathcal{H}_{\mathbf{i}^{\mathrm{o}^{-}}}^{\hbar}$ and $\mathcal{H}_{\mathbf{i}^{\prime}}^{\hbar} \otimes \mathcal{H}_{\mathbf{i}^{\prime}}^{\hbar}$-module structures. By this we mean only that the operator $\mathbf{K}_{\mathbf{i} \rightarrow \mathbf{i}^{\prime}}$ intertwines the action of the generators of $\mathcal{H}_{\mathbf{i}}^{\hbar} \otimes \mathcal{H}_{\mathbf{i}^{\circ}}^{\hbar}$ withthe action of their images under the gluing map map $\kappa_{|\mathbf{i}|}^{\hbar}\left(\mathbf{i} \rightarrow \mathbf{i}^{\prime}\right)$.

The function $G$. Let us introduce our key function

$$
\begin{gathered}
G\left(a_{1}, \ldots, a_{n}\right)= \\
\int \Phi^{\hbar_{k}}\left(\widehat{d}_{k} c-\sum_{j} \varepsilon_{k j} a_{j}\right)^{-1} \Phi^{\hbar_{k}}\left(-\widehat{d}_{k} c-\sum_{j} \varepsilon_{k j} a_{j}\right) \exp \left(\frac{\sum_{j \mid \varepsilon_{k j}<0} \varepsilon_{k j} a_{j}+a_{k}}{\pi i \hbar}\right) d c .
\end{gathered}
$$

Substituting the explicit integral expression for the function $\Phi^{\hbar_{k}}(z)$ one gets

$$
G\left(a_{1}, \ldots, a_{n}\right)=\int \exp \left(\int_{\Omega} \frac{\exp \left(i t \sum_{j} \varepsilon_{k j} a_{j}\right) \sin \left(t \widehat{d}_{k} c\right)}{2 i t \operatorname{sh}(\pi t) \operatorname{sh}\left(\pi \hbar_{k} t\right)} d t+c \frac{\sum_{j \mid \varepsilon_{k j}<0} \varepsilon_{k j} a_{j}+a_{k}}{\pi i \hbar}\right) d c
$$

We denote by $\left(a_{1}, \ldots, a_{n}\right)$ the logarithmic coordinates corresponding to $v$, and by $\left(a_{1}, \ldots, a_{k}^{\prime}, \ldots, a_{n}\right)$ the ones corresponding to $\mathbf{i}^{\prime}$. Recall that only the coordinate $a_{k}$ changes under the mutation $\mu_{k}$. Let us define the operator $\mathbf{K}_{\mathbf{i} \rightarrow \mathbf{i}^{\prime}}$ by

$$
\left(\mathbf{K}_{\mathbf{i} \rightarrow \mathbf{i}^{\prime}} f\right)\left(a_{1}, \ldots, a_{k}^{\prime}, \ldots, a_{n}\right):=\int G\left(a_{1}, \ldots, a_{k}^{\prime}+a_{k}, \ldots, a_{n}\right) f\left(a_{1}, \ldots, a_{k}, \ldots, a_{n}\right) d a_{k},
$$

where $a_{k}^{\prime}+a_{k}$ and $a_{k}$ are on the $k$-th places.

Theorem 4.3 The operators $\mathbf{K}_{\mathbf{i} \rightarrow \mathbf{i}^{\prime}}$ intertwine the $\mathcal{H}_{\mathbf{i}}^{\hbar} \otimes \mathcal{H}_{\mathbf{i}^{\mathrm{o}}}^{\hbar}$-module structures on $L^{2}\left(\mathcal{A}_{\mathbf{i}}^{+}\right)$provided by Lemma 4.2. 
Remark. We prove in [FG3] that the collection of Hilbert space $L^{2}\left(\mathcal{A}_{\mathbf{i}}^{+}\right)$and operators $\mathbf{K}_{\mathbf{i} \rightarrow \mathbf{i}^{\prime}}$ provide a unitary projective representation of the groupoid $\mathcal{G}_{|\mathbf{i}|}$. This implies that the operators $\mathbf{K}_{\mathbf{i} \rightarrow \mathbf{i}^{\prime}}$ give rise to a unitary projective representation of the cluster modular group $\Gamma_{|\mathbf{i}|}$ in $L^{2}\left(\mathcal{A}_{\mathbf{i}}^{+}\right)$.

Proof. We present a computation which allows to find the function $G$ as a unique up to a scalar function such that the corresponding integral transformation intertwines the $\mathcal{H}_{\mathbf{i}^{-}}^{\hbar}$ and $\mathcal{H}_{\mathbf{i}^{\prime}}^{\hbar}$ bimodule structures on $L^{2}\left(\mathcal{A}_{\mathbf{i}}^{+}\right)$and $L^{2}\left(\mathcal{A}_{\mathbf{i}^{\prime}}^{+}\right)$. Recall that $\varepsilon_{i j}^{o}=-\varepsilon_{i j}$, so we may write $\varepsilon_{i j}^{ \pm}:= \pm \varepsilon_{i j}$ and denote by $x_{i}^{ \pm}$the $x_{i}$-coordinates for the seeds $\mathbf{i}$ and $\mathbf{i}^{\mathbf{o}}$.

So we have to find $G$ such that the integral transformation (15) induces a map of operators:

$$
\widehat{x}_{i}^{\prime \pm} \longmapsto \begin{cases}\widehat{x}_{i}^{ \pm}-\varepsilon_{i k}^{ \pm} \phi^{\hbar_{k}}\left(-\operatorname{sgn}\left(\varepsilon_{i k}^{ \pm}\right) \widehat{x}_{k}^{ \pm}\right) & \text {if } i \neq k \\ -\widehat{x}_{k}^{ \pm} & \text {if } i=k .\end{cases}
$$

This means that we should have (changing $\widehat{x}_{i}^{-}$to $-\widehat{x}_{i}^{-}$for convenience) for $i \neq k$ :

$$
\begin{gathered}
\pi i \hbar \widehat{d}_{i} \frac{\partial}{\partial a_{i}} \pm \sum_{j \neq k} \varepsilon_{i j}^{\prime} a_{j} \pm \varepsilon_{i k}^{\prime} a_{k}^{\prime} \mapsto \\
\mapsto \pi i \hbar \widehat{d}_{i} \frac{\partial}{\partial a_{i}} \pm \sum_{j \neq k} \varepsilon_{i j} a_{j} \pm \varepsilon_{i k}\left(a_{k}-\phi^{\hbar k}\left(-\operatorname{sgn}\left( \pm \varepsilon_{\mathrm{ik}}\right)\left(\pi \mathrm{i} \hbar \widehat{\mathrm{d}}_{\mathrm{k}} \frac{\partial}{\partial \mathrm{a}_{\mathrm{k}}} \pm \sum_{\mathrm{j}} \varepsilon_{\mathrm{kj}} \mathrm{a}_{\mathrm{j}}\right)\right)\right)
\end{gathered}
$$

and

$$
\pi i \hbar \widehat{d}_{k} \frac{\partial}{\partial a_{k}^{\prime}} \pm \sum_{j} \varepsilon_{k j}^{\prime} a_{j} \mapsto-\left(\pi i \hbar \widehat{d}_{k} \frac{\partial}{\partial a_{k}} \pm \sum_{j} \varepsilon_{k j} a_{j}\right) .
$$

Here we use the following conventions. The signs \pm in our formulas always use either + everywhere, or - everywhere, so $\mp:=- \pm$. Thus we have one set of the equations corresponding to the upper signs and another one to the lower signs.

Observe that $\varepsilon_{k k}=0$. The relation (18) is satisfied by (15) if and only if

$$
-\varepsilon_{k j}^{\prime}=\varepsilon_{k j}, \quad \text { and } \quad \frac{\partial}{\partial a_{k}^{\prime}} \longmapsto-\frac{\partial}{\partial a_{k}} \text {. }
$$

Since these two conditions are evidently valid, we have the relation (18).

Substituting (17) into (15) one gets the identities:

$$
\begin{gathered}
\int\left(\pi i \hbar \widehat{d}_{i} \frac{\partial G}{\partial a_{i}} f+\pi i \hbar \widehat{d}_{i} G \frac{\partial f}{\partial a_{i}} \pm \sum_{j \neq k} \varepsilon_{i j}^{\prime} a_{j} G f \pm \varepsilon_{i k}^{\prime} a_{k}^{\prime} G f\right) d a_{k}= \\
=\int \pi i \hbar \widehat{d}_{i} G \frac{\partial f}{\partial a_{i}} \pm \sum_{j \neq k} \varepsilon_{i j} a_{j} G f \pm G \varepsilon_{i k}\left(a_{k}-\phi^{\hbar k}\left(\operatorname{sgn}\left(\varepsilon_{\mathrm{ik}}\right)\left(\mp \pi \mathrm{i} \hbar \widehat{\mathrm{d}}_{\mathrm{k}} \frac{\partial}{\partial \mathrm{a}_{\mathrm{k}}}-\sum_{\mathrm{j}} \varepsilon_{\mathrm{kj}} \mathrm{a}_{\mathrm{j}}\right)\right) f\right) d a_{k} .
\end{gathered}
$$

Since these identities should be valid for any $f$ one gets the equations for the function $G$ :

$$
\left(\pi i \hbar \widehat{d}_{i} \frac{\partial}{\partial a_{i}} \pm \sum_{j \neq k}\left(\varepsilon_{i j}^{\prime}-\varepsilon_{i j}\right) a_{j} \mp \varepsilon_{i k} a_{k} \pm \varepsilon_{i k} \phi^{\hbar_{k}}\left(\operatorname{sgn}\left(\varepsilon_{\mathrm{ik}}\right)\left( \pm \pi \mathrm{i} \hbar \widehat{\mathrm{d}}_{\mathrm{k}} \frac{\partial}{\partial \mathrm{a}_{\mathrm{k}}}-\sum_{\mathrm{j}} \varepsilon_{\mathrm{kj}} \mathrm{a}_{\mathrm{j}}\right)\right)\right) G=0
$$


Let us introduce the function $\widehat{G}$ related to $G$ by the Fourier transform:

$$
\widehat{G}(c)=\int e^{-\frac{a_{k} c}{\pi i \hbar}} G\left(a_{k}\right) d a_{k} ; \quad G\left(a_{k}\right)=\frac{1}{2 \pi^{2} \hbar} \int e^{\frac{a_{k} c}{\pi i \hbar}} \widehat{G}(c) d c
$$

(we omit the variables $a_{1}, \ldots, a_{k-1}, a_{k+1}, \ldots, \ldots, a_{n}$ both $G$ and $\widehat{G}$ depends on). Taking into account the relations

$$
\pi i \hbar \frac{\widehat{\partial G}}{\partial a_{k}}=c \widehat{G}, \widehat{a_{k} G}=-\pi i \hbar \frac{\partial \widehat{G}}{\partial c}
$$

one can get the equation for the function $\widehat{G}$ :

$$
\left(\pi i \hbar \widehat{d}_{i} \frac{\partial}{\partial a_{i}} \pm \sum_{j \neq k}\left(\varepsilon_{i j}^{\prime}-\varepsilon_{i j}\right) a_{j} \pm \varepsilon_{i k} \pi i \hbar \frac{\partial}{\partial c} \pm \varepsilon_{i k} \phi^{\hbar_{k}}\left(\operatorname{sgn}\left(\varepsilon_{\mathrm{ik}}\right)\left( \pm \widehat{\mathrm{d}}_{\mathrm{k}} \mathrm{c}-\sum_{\mathrm{j}} \varepsilon_{\mathrm{kj}} \mathrm{a}_{\mathrm{j}}\right)\right) \widehat{G}=0 .\right.
$$

Taking sum and difference of the equations corresponding to the upper and lower signs one obtains:

$$
\left(2 \pi i \hbar \widehat{d}_{i} \frac{\partial}{\partial a_{i}}+\varepsilon_{i k} \phi^{\hbar_{k}}\left(\operatorname{sgn}\left(\varepsilon_{\mathrm{ik}}\right)\left(\widehat{\mathrm{d}}_{\mathrm{k}} \mathrm{c}-\sum_{\mathrm{j}} \varepsilon_{\mathrm{kj}} \mathrm{a}_{\mathrm{j}}\right)\right)-\varepsilon_{i k} \phi^{\hbar_{k}}\left(\operatorname{sgn}\left(\varepsilon_{\mathrm{ik}}\right)\left(-\widehat{\mathrm{d}}_{\mathrm{k}} \mathrm{c}-\sum_{\mathrm{j}} \varepsilon_{\mathrm{kj}} \mathrm{a}_{\mathrm{j}}\right)\right) \widehat{G}=0\right.
$$

and

$$
\begin{gathered}
\left(2 \sum_{j \neq k}\left(\varepsilon_{i j}^{\prime}-\varepsilon_{i j}\right) a_{j}+2 \varepsilon_{i k} \pi i h \frac{\partial}{\partial c}+\right. \\
\left.+\varepsilon_{i k} \phi^{\hbar_{k}}\left(\operatorname{sgn}\left(\varepsilon_{\mathrm{ik}}\right)\left(\widehat{\mathrm{d}}_{\mathrm{k}} \mathrm{c}-\sum_{\mathrm{j}} \varepsilon_{\mathrm{kj}} \mathrm{a}_{\mathrm{j}}\right)\right)+\varepsilon_{i k} \phi^{\hbar_{k}}\left(\operatorname{sgn}\left(\varepsilon_{\mathrm{ik}}\right)\left(-\widehat{\mathrm{d}}_{\mathrm{k}} \mathrm{c}-\sum_{\mathrm{j}} \varepsilon_{\mathrm{kj}} \mathrm{a}_{\mathrm{j}}\right)\right)\right) \widehat{G}=0 .
\end{gathered}
$$

Observe that these are the system of $2 n-2$ equations on a function of $n$ variables. So it is an overdetermined system if $n>2$. Using the identities

$$
\phi^{\hbar_{k}}(\operatorname{sgn}(\mathrm{a}) \mathrm{b})=\phi^{\hbar_{\mathrm{k}}}(\mathrm{b})+(\operatorname{sgn}(\mathrm{a})-1) \mathrm{b} / 2 ; \quad \widehat{\mathrm{d}}_{\mathrm{i}} \varepsilon_{\mathrm{ji}}=-\widehat{\mathrm{d}}_{\mathrm{j}} \varepsilon_{\mathrm{ij}}
$$

they can be transformed to the form

$$
2 \pi i \hbar \frac{\partial \log \widehat{G}}{\partial a_{i}}=\widehat{d}_{k}^{-1} \varepsilon_{k i}\left(\phi^{\hbar_{k}}\left(\widehat{d}_{k} c-\sum_{j} \varepsilon_{k j} a_{j}\right)-\phi^{\hbar_{k}}\left(-\widehat{d}_{k} c-\sum_{j} \varepsilon_{k j} a_{j}\right)+\widehat{d}_{k} c\left(\operatorname{sgn}\left(\varepsilon_{\mathrm{ik}}\right)-1\right)\right)
$$

and

$$
\begin{gathered}
2 \pi i \hbar \frac{\partial \log \widehat{G}}{\partial c}=2\left(\varepsilon_{i k}\right)^{-1} \sum_{j \neq k}\left(\varepsilon_{i j}-\varepsilon_{i j}^{\prime}\right) a_{j} \\
-\phi^{\hbar_{k}}\left(\widehat{d}_{k} c-\sum_{j} \varepsilon_{k j} a_{j}\right)-\phi^{\hbar_{k}}\left(-\widehat{d}_{k} c-\sum_{j} \varepsilon_{k j} a_{j}\right)+\left(\operatorname{sgn}\left(\varepsilon_{\mathrm{ik}}\right)-1\right) \sum_{\mathrm{j}} \varepsilon_{\mathrm{kj}} \mathrm{a}_{\mathrm{j}}
\end{gathered}
$$

Taking into account that

$$
\varepsilon_{i j}^{\prime}-\varepsilon_{i j}=\frac{\left|\varepsilon_{i k}\right| \varepsilon_{k j}+\varepsilon_{i k}\left|\varepsilon_{k j}\right|}{2}, \quad i, j \neq k
$$


we have the following identity:

$$
2\left(\varepsilon_{i k}\right)^{-1}\left(\varepsilon_{i j}-\varepsilon_{i j}^{\prime}\right)+\left(\operatorname{sgn}\left(\varepsilon_{\mathrm{ik}}\right)-1\right) \varepsilon_{\mathrm{kj}}=\left(\operatorname{sgn}\left(\varepsilon_{\mathrm{jk}}\right)-1\right) \varepsilon_{\mathrm{kj}}
$$

Multiplying it by $a_{j}$ and taking the sum over $j \neq k$ we get

$$
2\left(\varepsilon_{i k}\right)^{-1} \sum_{j \neq k}\left(\varepsilon_{i j}-\varepsilon_{i j}^{\prime}\right) a_{j}+\left(\operatorname{sgn}\left(\varepsilon_{\mathrm{ik}}\right)-1\right) \sum_{\mathrm{j}} \varepsilon_{\mathrm{kj}} \mathrm{a}_{\mathrm{j}}=\sum_{\mathrm{j}}\left(\operatorname{sgn}\left(\varepsilon_{\mathrm{jk}}\right)-1\right) \varepsilon_{\mathrm{kj}} \mathrm{a}_{\mathrm{j}},
$$

Thus (21) is equivalent to

$$
2 \pi i \hbar \frac{\partial \log \widehat{G}}{\partial c}=-\phi^{\hbar_{k}}\left(\widehat{d}_{k} c-\sum_{j} \varepsilon_{k j} a_{j}\right)-\phi^{\hbar_{k}}\left(-\widehat{d}_{k} c-\sum_{j} \varepsilon_{k j} a_{j}\right)+\left(\operatorname{sgn}\left(\varepsilon_{\mathrm{jk}}\right)-1\right) \sum_{\mathrm{j}} \varepsilon_{\mathrm{kj}} \mathrm{a}_{\mathrm{j}}
$$

Therefore the solution of the equations (20) and (21) is given by the formula

$$
\widehat{G}=C \Phi^{\hbar_{k}}\left(\widehat{d}_{k} c-\sum_{j} \varepsilon_{k j} a_{j}\right)^{-1} \Phi^{\hbar_{k}}\left(-\widehat{d}_{k} c-\sum_{j} \varepsilon_{k j} a_{j}\right) e^{\left.c \sum_{j}\left(\operatorname{sgn}\left(\varepsilon_{\mathrm{jk}}\right)-1\right)\right) \varepsilon_{\mathrm{kj}} \mathrm{a}_{\mathrm{j}} / 2 \pi \mathrm{i} \hbar},
$$

where $C$ is an arbitrary constant. Taking $C=2 \pi^{2} \hbar$, one obtains the desired formula (14). The statement is proved.

Lemma 4.4 An integral operator given by the formula (15) for certain function $G$ intertwines the operators (16) if and only if the standard formula for the mutation of the function $\varepsilon_{i j}$ holds.

Proof. The proof of the theorem shows that this formula, as well as the formula for mutations of the $A$-coordinates follow from the anzatz (15) and the mutation formulas for the quantized $X$-coordinates.

Representation of the modular double of the chiral double of $\mathcal{X}_{|\mathbf{i}|, q}$. Combining Claim 3.6 and Theorem 4.3 we see that the collection of Hilbert spaces $\left\{L_{2}\left(\mathcal{A}_{\mathbf{i}}^{+}\right)\right\}$should provide a projective unitary *-representation of the modular double of the chiral double of $\mathcal{X}_{|\mathbf{i}|, q}$, defined as

$$
\mathcal{X}_{|\mathbf{i}|, q} \times \mathcal{X}_{\left|\mathbf{i}^{\circ}\right|, q} \times \mathcal{X}_{\left|\mathbf{i}^{\vee}\right|, q^{\vee}} \times \mathcal{X}_{\mid \mathbf{i}^{\circ} \vee, q^{\vee}}
$$

According to [FG3], the collection of Hilbert spaces $\left\{L_{2}\left(\mathcal{A}_{\mathbf{i}}^{+}\right)\right\}$should provide a representation of the modular double $\mathcal{D}_{|\mathbf{i}|, q} \times \mathcal{D}_{|\mathbf{i} \vee|, q}$ of the cluster double of the quantum cluster $\mathcal{X}$-variety $\mathcal{X}_{|\mathbf{i}|, q}$. Since there is a canonical map of quantum spaces $\mathcal{D}_{|\mathbf{i}|, q} \longrightarrow \mathcal{X}_{|\mathbf{i}|, q} \times \mathcal{X}_{\left|\mathbf{i}^{\circ}\right|, q}$, this implies the above claim.

\section{The quantum logarithm and dilogarithm functions}

The proofs of all results listed above can be found in [FG3].

Recall the dilogarithm function

$$
\operatorname{Li}_{2}(x):=-\int_{0}^{x} \log (1-t) d t
$$

The quantum logarithm function. It is the following function:

$$
\phi^{\hbar}(z):=-2 \pi \hbar \int_{\Omega} \frac{e^{-i p z}}{\left(e^{\pi p}-e^{-\pi p}\right)\left(e^{\pi \hbar p}-e^{-\pi \hbar p}\right)} d p ;
$$

where the contour $\Omega$ goes along the real axes from $-\infty$ to $\infty$ bypassing the origin from above. 
Proposition 5.1 The function $\phi^{\hbar}(x)$ enjoys the following properties.

$$
\begin{gathered}
\lim _{\hbar \rightarrow 0} \phi^{\hbar}(z)=\log \left(e^{z}+1\right) . \\
\phi^{\hbar}(z)-\phi^{\hbar}(-z)=z . \\
\overline{\phi^{\hbar}(z)}=\phi^{\hbar}(\bar{z}) . \\
\phi^{\hbar}(z) / \hbar=\phi^{1 / \hbar}(z / \hbar) . \\
\phi^{\hbar}(z+i \pi \hbar)-\phi^{\hbar}(z-i \pi \hbar)=\frac{2 \pi i \hbar}{e^{-z}+1}, \quad \phi^{\hbar}(z+i \pi)-\phi^{\hbar}(z-i \pi)=\frac{2 \pi i}{e^{-z / \hbar}+1} .
\end{gathered}
$$

(A6) The form $\phi^{\hbar}(z) d z$ is meromorphic with poles at the points $\{\pi i((2 m-1)+(2 n-1) \hbar) \mid m, n \in \mathbb{N}\}$ with residues $2 \pi i \hbar$ and at the points $\{-\pi i((2 m-1)+(2 n-1) \hbar) \mid m, n \in \mathbb{N}\}$ with residues $-2 \pi i \hbar$.

The quantum dilogarithm. Recall the quantum dilogarithm function:

$$
\Phi^{\hbar}(z):=\exp \left(-\frac{1}{4} \int_{\Omega} \frac{e^{-i p z}}{\operatorname{sh}(\pi p) \operatorname{sh}(\pi \hbar p)} \frac{d p}{p}\right) .
$$

It goes back to Barnes [Ba], and was used by Baxter [Bax], Faddeeev [Fad], and others.

Proposition 5.2 The function $\Phi^{\hbar}(x)$ enjoys the following properties.

$$
2 \pi i \hbar d \log \Phi^{\hbar}(z)=\phi^{\hbar}(z) d z
$$

$$
\lim _{\Re z \rightarrow-\infty} \Phi^{\hbar}(z)=1
$$

Here the limit is taken along a line parallel to the real axis.

$$
\begin{gathered}
\lim _{\hbar \rightarrow 0} \Phi^{\hbar}(z) / \exp \frac{-L i_{2}\left(-e^{z}\right)}{2 \pi i \hbar}=1 . \\
\Phi^{\hbar}(z) \Phi^{\hbar}(-z)=\exp \left(\frac{z^{2}}{4 \pi i \hbar}\right) e^{-\frac{\pi i}{12}\left(\hbar+\hbar^{-1}\right)} . \\
\overline{\Phi^{\hbar}(z)}=\left(\Phi^{\hbar}(\bar{z})\right)^{-1} . \text { In particular }\left|\Phi^{\hbar}(z)\right|=1 \text { for } z \in \mathbb{R} . \\
\Phi^{\hbar}(z)=\Phi^{1 / \hbar}(z / \hbar) . \\
\Phi^{\hbar}(z+2 \pi i \hbar)=\Phi^{\hbar}(z)\left(1+q e^{z}\right), \quad \Phi^{\hbar}(z+2 \pi i)=\Phi^{\hbar}(z)\left(1+q^{\vee} e^{z / \hbar}\right) .
\end{gathered}
$$

(B6) The function $\Phi^{\hbar}(z) d z$ is meromorphic with poles at the points

$$
\{\pi i((2 m-1)+(2 n-1) \hbar) \mid m, n \in \mathbb{N}\}
$$

and zeroes at the points

$$
\{-\pi i((2 m-1)+(2 n-1) \hbar) \mid m, n \in \mathbb{N}\}
$$




\section{References}

[Ba] Barnes E.W.: The genesis of the double gamma function. Proc. London Math. Soc. 31 (1899) 358-381.

[Bax] Baxter R.: Exactly solved models in statistical mechanics. Academic Press (1982).

[BFZ] Berenstein A., Fomin S., Zelevinsky A.: Cluster algebras III: Upper bounds and double Bruhat cells. math.RT/0305434. J. Amer. Math. Soc. 15 (2002), no. 2, 497-529

[Fad] Faddeev L.D. Discrete Heisenberg-Weyl group and modular group. Lett. Math. Phys. 34 (1995), no. 3, 249-254.

[FCh] Fock, V. V.; Chekhov, L. O.: Quantum Teichmüller spaces. Teoret. Mat. Fiz. 120 (1999), no. $3,511-528$.

[FG1] Fock V.V., Goncharov A.B. Moduli spaces of local systems and higher Teichmuller theory. Publ. Math. IHES, n. 103 (2006) 1-212. ArXiv math.AG/0311149.

[FG2] Fock V.V., Goncharov A.B. Cluster ensembles, quantization and the dilogarithm I. ArXiv math.AG/0311245.

[FG3] Fock V.V., Goncharov A.B. The quantum dilogarithm and unitary representations of the modular groupoid. math.QA/0702397.

[FZI] Fomin S., Zelevinsky A.: Cluster algebras. I: Foundations. J. Amer. Math. Soc. 15 (2002), no. $2,497-529$

[K] Kashaev R.M.: Quantization of Teichmüller spaces and the quantum dilogarithm. Lett. Math. Phys. 43 (1998), no. 2, 105-115.

A.G.: Department of Math, Brown University, Providence RI 02906, USA; sasha@math.brown.edu V.F.: ITEP, B. Cheremushkinskaya 25, 117259 Moscow, Russia. fock@math.brown.edu 